\title{
MONOTONICITY OF FUNCTIONS AND SIGN CHANGES OF THEIR CAPUTO DERIVATIVES
}

\author{
Kai Diethelm ${ }^{1,2}$
}

\begin{abstract}
It is well known that a continuously differentiable function is monotone in an interval $[a, b]$ if and only if its first derivative does not change its sign there. We prove that this is equivalent to requiring that the Caputo derivatives of all orders $\alpha \in(0,1)$ with starting point $a$ of this function do not have a change of sign there. In contrast to what is occasionally conjectured, it not sufficient if the Caputo derivatives have a constant sign for a few values of $\alpha \in(0,1)$ only.
\end{abstract}

MSC 2010: Primary 26A33

Key Words and Phrases: fractional calculus, Caputo derivative, monotone function, sign change.

\section{Introduction}

Classical integer order calculus provides numerous results that link the shape properties of a function's graph (such as, e. g., monotonicity or the location of extrema) with the signs of its first or second derivative. The following theorems contain some specific examples; since they are so well known, we omit the proofs.

Theorem 1.1. A function $f \in C^{1}[a, b]$ is monotone on $[a, b]$ if and only if its first derivative $f^{\prime}$ does not change its sign in this interval.

October 12, 2015 
TheOREM 1.2. If a function $f \in C^{1}[a, b]$ attains a global maximum or a global minimum at some point $t^{*} \in(a, b)$ then $f^{\prime}\left(t^{*}\right)=0$.

If a function $f \in C^{2}[a, b]$ attains a global maximum at some point $t^{*} \in(a, b)$ then $f^{\prime}\left(t^{*}\right)=0$ and $f^{\prime \prime}\left(t^{*}\right) \leq 0$; if $t^{*} \in(a, b)$ is the location of a global minimum of $f$ then $f^{\prime}\left(t^{*}\right)=0$ and $f^{\prime \prime}\left(t^{*}\right) \geq 0$.

It is quite natural to ask for generalizations of such statements that involve fractional, rather than integer order, derivatives. In this paper we concentrate on fractional derivatives of Caputo type [2, Chapter 3], i. e.

$$
D_{* a}^{\alpha} f(t):=J_{a}^{\lceil\alpha\rceil-\alpha} D^{\lceil\alpha\rceil} f(t)
$$

for $\alpha>0$ and $\alpha \notin \mathbb{N}$ where $\lceil\cdot\rceil$ denotes the ceiling function, $D^{m}$ is the standard $m$ th order differential operator for $m \in \mathbb{N}$ and $J_{a}^{\beta}$ is the RiemannLiouville integral operator of order $\beta>0$ with starting point $a$, viz.

$$
J_{a}^{\beta} g(t):=\frac{1}{\Gamma(\beta)} \int_{a}^{t}(t-s)^{\beta-1} g(s) \mathrm{d} s .
$$

In this case, the following fractional extension of the first part of Theorem 1.2 has been shown by Luchko [4, Theorem 1]:

TheOREM 1.3. If a function $f \in C^{1}[a, b]$ attains a global maximum at some point $t^{*} \in(a, b)$ then $D_{* a}^{\alpha} f\left(t^{*}\right) \geq 0$ for all $\alpha \in(0,1)$. If $t^{*} \in(a, b)$ is the location of the global minimum of $f$ then $D_{* a}^{\alpha} f\left(t^{*}\right) \leq 0$ for all $\alpha \in(0,1)$.

In fact, Luchko's result is more general than our formulation here since he admits functions $f$ from a slightly larger class of functions; however, for the sake of simplicity, we have chosen this marginally restricted version.

Some authors have believed that this result can be extended to fractional derivatives of order $\alpha \in(1,2)$; specifically they claimed that, under the additional condition $f \in C^{2}[a, b]$, the inequality

$$
D_{* a}^{\alpha} f\left(t^{*}\right) \geq 0
$$

holds for these $\alpha$ if $t^{*}$ is a global minimum point of $f$. While Luchko's result (Theorem 1.3) extends the first part of the classical Theorem 1.2. such a result would provide an extension of the second part of Theorem $\mathbf{1 . 2}$. However, recently Al-Refai [1] (who also provides some references to claims of such results in the literature) explicitly constructed a counterexample and hence demonstrated that the second part of Theorem $\mathbf{1 . 2}$ cannot be extended in this manner.

Our goal here is to perform a similar investigation for possible fractional versions of Theorem 1.1. In a certain sense, it thus continues the author's earlier work [3] that transferred other classical calculus results, 
including the mean value theorems of differential and integral calculus, to the fractional setting.

\section{Caputo derivatives of monotone functions}

We begin our investigation of a generalization of Theorem $\mathbf{1 . 1}$ to the fractional case with a very simple but nevertheless useful observation.

Theorem 2.1. Let the function $f \in C^{1}[a, b]$ be monotone on $[a, b]$. Then, for all $\alpha \in(0,1)$ and all $t \in[a, b]$, we have that

$$
D_{* a}^{\alpha} f(t) \begin{cases}\leq 0 & \text { if } f \text { is decreasing, } \\ \geq 0 & \text { if } f \text { is increasing. }\end{cases}
$$

In this formulation, we use the terms "increasing" and "decreasing" in the non-strict sense.

$\mathrm{P}$ r o o $\mathrm{f}$. Assume that $f$ is increasing. Then, $f^{\prime}$ is nonnegative in $[a, b]$, and hence, by the definition of the Caputo operator given in eq. (1.1) and eq. (1.2),

$$
D_{* a}^{\alpha} f(t)=\frac{1}{\Gamma(1-\alpha)} \int_{a}^{t}(t-s)^{-\alpha} f^{\prime}(s) \mathrm{d} s \geq 0 .
$$

In the case of a decreasing function $f$, we argue in an analog way.

We obtain an immediate consequence that can be interpreted as the fractional generalization of the "forward direction" of Theorem 1.1.

Corollary 2.1. Let the function $f \in C^{1}[a, b]$ be monotone on $[a, b]$. Then, for all $\alpha \in(0,1)$, the Caputo derivative $D_{* a}^{\alpha} f$ does not have a change of sign in $[a, b]$.

But we can also obtain a fractional generalization of the "backward direction" of Theorem 1.1.

TheOREM 2.2. Assume that $f \in C^{1}[a, b]$ is such that $D_{* a}^{\alpha} f(t) \geq 0$ for all $t \in[a, b]$ and all $\alpha \in\left(\alpha_{0}, 1\right)$ with some $\alpha_{0} \in(0,1)$. Then, $f$ is monotone increasing. Similarly, if $D_{* a}^{\alpha} f(t) \leq 0$ for all $t$ and $\alpha$ mentioned above, then $f$ is monotone decreasing.

P r o o f. Our observation in the first case is based on the definition of the Caputo derivative and certain well known properties of the RiemannLiouville integral. Specifically, consider first the case that $t \in(a, b]$. Then, 
in view of eq. (1.1) and our assumption, $0 \leq D_{* a}^{\alpha} f(t)=J_{a}^{1-\alpha} f^{\prime}(t)$. Since $f^{\prime}$ is assumed to be continuous, we may invoke [2, Theorem 2.10] to determine the limit $\alpha \rightarrow 1-$ of the right-hand side of this inequality; this yields

$$
0 \leq \lim _{\alpha \rightarrow 1-} D_{* a}^{\alpha} f(t)=\lim _{\alpha \rightarrow 1-} J_{a}^{1-\alpha} f^{\prime}(t)=f^{\prime}(t) .
$$

We thus conclude that $f^{\prime}$ is nonnegative on the half-open interval $(a, b]$. The continuity of $f^{\prime}$ on the closed interval $[a, b]$ then implies that $f^{\prime}(a) \geq 0$ as well; hence $f^{\prime}$ is nonnegative on the entire interval $[a, b]$, and it follows that $f$ is increasing.

The second claim follows immediately if we apply the result that we have just shown to $-f$ instead of $f$.

Theorem 2.2 has an interesting consequence that we explicitly note.

Theorem 2.3. Assume that $f \in C^{1}[a, b]$ is such that $D_{* a}^{\alpha} f(t) \geq 0$ for all $t \in[a, b]$ and all $\alpha \in\left(\alpha_{0}, 1\right)$ with some $\alpha_{0} \in(0,1)$. Then, $D_{* a}^{\alpha} f(t) \geq 0$ for all $t \in[a, b]$ and all $\alpha \in(0,1)$.

The analog statement holds when the sign of the derivatives is reversed.

Thus, if we can prove that the Caputo derivatives of $f$ are of a constant sign for all $\alpha$ sufficiently close to 1 , then it already follows that this property even holds for all $\alpha$ from the complete interval $(0,1)$ and, by Theorem $\mathbf{2 . 2}$. also for the classical case $\alpha=1$.

P r o o f. If the Caputo derivatives $D_{* a}^{\alpha} f(t)$ are nonnegative for the indicated values of $t$ and $\alpha$ then Theorem $\mathbf{2 . 2}$ tells us that $f$ is increasing; the claim then follows from Theorem 2.1. The statement for the case of opposite signs of $D_{* a}^{\alpha} f(t)$ follows in an analog manner.

It thus turns out that, contrary to an occasionally stated conjecture, the lack of a change of sign of $D_{* a}^{\alpha} f$ for some, but not all, $\alpha \in(0,1)$ is not sufficient to imply the monotonicity of $f$. One may ask how a non-monotone functions which has a fractional derivative without a change of sign may look like, and in particular whether such a function must necessarily be of a very exotic or even pathological nature. The following example demonstrates that this is not the case; indeed a simple polynomial of a quite low degree can already have this property. An interesting aspect in this connection is the fact that our counterexample is the same one that Al-Refai used in his investigations from [1, Section 2] regarding the fractional version of the second part of Theorem $\mathbf{1 . 2}$ that we mentioned above. 
Example 2.1. Define the function $g$ by

$$
g(t):=t\left(t-\frac{1}{2}\right)(t-1)=t^{3}-\frac{3}{2} t^{2}+\frac{1}{2} t,
$$

so that

$$
g^{\prime}(t)=3 t^{2}-3 t+\frac{1}{2}
$$

and

$$
\begin{aligned}
D_{* 0}^{\alpha} g(t) & =\frac{6}{\Gamma(4-\alpha)} t^{3-\alpha}-\frac{3}{\Gamma(3-\alpha)} t^{2-\alpha}+\frac{1}{2 \Gamma(2-\alpha)} t^{1-\alpha} \\
& =\frac{6 t^{1-\alpha}}{\Gamma(4-\alpha)}\left(t^{2}-\frac{1}{2}(3-\alpha) t+\frac{1}{12}(3-\alpha)(2-\alpha)\right)
\end{aligned}
$$

for $0<\alpha<1$. It follows from eq. (2.2) that the only zeros of $D_{* 0}^{\alpha} g$ are located at $t_{1}(\alpha)=0, t_{2}(\alpha)=(3-\alpha-\sqrt{(3-\alpha)(1+\alpha) / 3}) / 4$ and $t_{3}(\alpha)=(3-\alpha+\sqrt{(3-\alpha)(1+\alpha) / 3}) / 4$. Clearly, $t_{1}(\alpha)<t_{2}(\alpha) \leq t_{3}(\alpha)$, and hence we conclude that $D_{* 0}^{\alpha} g$ does not have a change of sign on $\left[0, t_{2}(\alpha)\right]$.

But now,

$$
\frac{\mathrm{d}}{\mathrm{d} \alpha} t_{2}(\alpha)=\frac{1}{4}\left(-1-\frac{1-\alpha}{\sqrt{3(3-\alpha)(1+\alpha)}}\right)<0
$$

for $0<\alpha<1$. Therefore, if we denote by $I_{\alpha}:=\left[0, t_{2}(\alpha)\right]$ the largest interval that has the left end point at 0 and that is such that $D_{* 0}^{\alpha} g$ does not have a change of sign on $I_{\alpha}$, then these intervals monotonically shrink as $\alpha$ increases. Specifically,

$$
\frac{1}{2}=t_{2}(0)>t_{2}\left(\alpha_{1}\right)>t_{2}\left(\alpha_{2}\right)>t_{2}(1)=\frac{3-\sqrt{3}}{6} \approx 0.211325,
$$

for $0<\alpha_{1}<\alpha_{2}<1$, and hence

$$
\left[0, \frac{1}{2}\right]=I_{0} \supset I_{\alpha_{1}} \supset I_{\alpha_{2}} \supset I_{1}=\left[0, \frac{3-\sqrt{3}}{6}\right] .
$$

Therefore, if we pick some $\alpha^{*} \in(0,1)$, then we can see that $D_{* 0}^{\alpha^{*}} g$ is nonnegative throughout the interval $I^{*}:=I_{\alpha^{*}}=\left[0, t_{2}\left(\alpha^{*}\right)\right]$. Moreover, it follows from our monotonicity observation on $t_{2}$ that all derivatives $D_{* 0}^{\alpha} g$ with $\alpha \in\left(0, \alpha^{*}\right)$ are nonnegative on the interval $I^{*}$ as well. Nevertheless, an elementary computation reveals that the only zeros of $g^{\prime}$ are located at $\tau_{1}=(3-\sqrt{3}) / 6=t_{2}(1)$ and $\tau_{2}=(3+\sqrt{3}) / 6=1-\tau_{1} \approx 0.788675$. From this observation and the fact that $g$ is a polynomial of degree 3 with a positive leading coefficient, we conclude that $g$ has a local maximum at the point $t_{2}(1)$ which is located in the interior of our interval $I^{*}$; hence, in spite 
of the fact that $D_{* 0}^{\alpha} g(t)>0$ for almost all $t \in I^{*}$ (with the sole exception of the point $t=0$ for which $D_{* 0}^{\alpha} g(t)=0$ holds) and all $\alpha \in\left(0, \alpha^{*}\right]$, the function $g$ is not monotone on $I^{*}$.

REMARK 2.1. We point out that all our investigations are based on the fact that the left end point of the interval on which the monotonicity is discussed coincides with the starting point of the Caputo differential operator. This is a very natural requirement because of the non-locality of the fractional differential operator. Specifically, if we had $a<a^{\prime}<b$ and wanted to find out whether a function $f$ is monotonic on $\left[a^{\prime}, b\right]$, then a criterion based on the behaviour of $D_{* a}^{\alpha} f$ would not be of any help since the values of $D_{* a}^{\alpha} f$ may de influenced to an arbitrarily large extent by the behaviour of $f$ on the interval $\left[a, a^{\prime}\right]$, i. e. outside of the interval in which we are interested. Therefore, in a situation like this only integer-order derivatives can be of any use because they are local operators and hence do not depend on the behaviour of $f$ outside of the interval of interest.

\section{References}

[1] M. Al-Refai, On the fractional derivatives at extreme points. Electron. J. Qual. Theory Differ. Equ. (2012), Paper No. 55.

[2] K. Diethelm, Fractional Differential Equations. Springer, Berlin (2010).

[3] K. Diethelm, The mean value theorems and a Nagumo-type uniqueness theorem for Caputo's fractional calculus. Fract. Calc. Appl. Anal. 15, No. 2 (2012), 304-313.

[4] Y. Luchko, Maximum principle for the generalized time-fractional diffusion equation. J. Math. Anal. Appl. 351 (2009), 218-223.

${ }^{1}$ AG Numerik

Institut Computational Mathematics

Technische Universität Braunschweig

Pockelsstr. 14

38106 Braunschweig, GERMANY

e-mail:k.diethelm@tu-bs.de

${ }^{2}$ GNS Gesellschaft für numerische Simulation mbH

Am Gaußberg 2

38114 Braunschweig, GERMANY

e-mail: diethelm@gns-mbh.com 